\title{
Dos supuestos testimonios del vino de Saguntum en Juvenal y en Frontón
}

\author{
Ricardo Hernández Pérez \\ Universidad de Valencia \\ ricardo.hernandez@uv.es \\ ORCID iD: http://orcid.org/0000-0002-5983-9375
}

\section{Two supposed testimonies of Saguntum wine in Iuuenalis and Fronto}

\begin{abstract}
Es muy probable que Iuu. V 24-49 y Fronto 134.23 ss. van den Hout ( $2^{\mathrm{a}}$ ed.) no se refieran, como se ha creído, al vino de Saguntum. Juvenal parece referirse a la cerámica saguntina, cuya baja calidad era proverbial en la tradición epigramática y satírica. El pasaje de Frontón sería un ejemplo más de la confusión, en la tradición manuscrita, entre Saguntum y Zacynthos y entre Saguntinus y Zacynthius.
\end{abstract}

Palabras clave: Juvenal; Frontón; Saguntum; Zacynthos; vino; lagona.
Although it has been believed Iuuenal V 24-49 and Fronto 134.23 ss. van den Hout (2nd ed.) do not probably refer to the Saguntum wine. Iuuenal appears to allude to Saguntine pottery, which proverbially was of low quality in the epigrammatic and satirical tradition. Fronto's sentence would be one more example of confusion in the manuscript tradition between Saguntum and Zacynthos on the one hand, and Saguntinus and Zacynthius on the other.

Keywords: Juvenal; Fronto; Saguntum; Zacynthos; wine; lagona.

Cómo citar este artículo / Citation: Hernández Pérez, Ricardo 2017: «Dos supuestos testimonios del vino de Saguntum en Juvenal y en Frontón», Emerita 85 (1), pp. 141-152.

Dos pasajes de la literatura latina del siglo II d. C. -pertenecientes a una sátira de Juvenal (V 24-29) y a una carta de Frontón (134.23 ss. van den Hout 1988²) y recogidos como fuentes de la Hispania romana en FHA VIII, pp. 279 s., 299 s. y en THA III, pp. 326 s., 340- se han considerado como referidos al vino de la antigua Saguntum y se han aducido como testimonios que confirmarían, no sólo que dicho vino se exportaba a la ciudad de Roma como el testimonio de la arqueología demuestra (es especialmente revelador el hallazgo de una misma marca anfórica que contiene el topónimo en ablativo Sacynto $=$ Sagunto 
tanto en el territorio de Saguntum -CIL II 62549- como en Roma -CIL XV $2632-)^{1}$, sino que además se conocía allí con su propia denominación de origen: uinum Saguntinum; como dice Aranegui 2004, p. 207 s., «se comprueba que entre los alrededores del año 80 y el $160 \mathrm{~d}$. C. la denominación saguntino para un vino común era utilizada por parte de escritores latinos» ${ }^{2}$. Ahora bien, creo que ambos pasajes, si se examinan atentamente, no pueden ser tenidos por testimonios absolutamente válidos al respecto. Veamos primero, por seguir un orden cronológico, Iuu. V 24-29:

Qualis cena tamen! uinum quod sucida nolit lana pati: de conuiua Corybanta uidebis.

iurgia proludunt, sed mox et pocula torques

saucius et rubra deterges uulnera mappa, inter uos quoties libertorumque cohortem pugna Saguntina feruet commissa lagona.

¡Pero vaya cena! Un vino que ni la lana sucia se avendría a soportar. Verás a los convidados convertidos en coribantes. Los insultos son el preludio, pero enseguida te pones a blandir borracho las copas y te limpias las heridas con una servilleta roja de sangre, cada vez que entre vosotros y la cohorte de libertos se entabla una bulliciosa batalla a golpes de garrafas saguntinas.

Estos versos son el inicio de la descripción de un banquete en el que el anfitrión humilla a sus clientes ofreciéndoles bebida, comida y vajilla de ínfima calidad, en contraste con las exquisiteces que él se reserva para sí mismo. Se dice, en primer lugar, que el vino servido a dichos clientes es de la peor calidad; para lo cual se recurre a una muy expresiva hipérbole que da a entender que era incluso peor que el vinagre (v. 24 s.: ... uinum quod sucida nolit / lana pati...). $)^{3}$. Hasta aquí, es evidente que Juvenal no indica el origen de dicho vino. A continuación relata, parodiando la dicción épica, cómo los

\footnotetext{
${ }^{2}$ Cf. también Aranegui 1990, p. 16; 1991, pp. 50-52; 1992, p. 37; 1999, p. 95; 2007, p. 53 s. En el mismo sentido, cf. Cisneros 2002, p. 131 s.

${ }^{3}$ La lana sucida (la lana recién esquilada y que, por tanto, tiene aún la mugre de la oveja) se empleaba, empapada en vino o vinagre y en aceite, como un remedio de aplicación externa (fomentum) en la medicina antigua (cf. Cels. II 33; Plin., Nat. XXIX 30-31). El vino empleado para ello no era, obviamente, de buena calidad. Cf. comm. ad loc. de Duff 1957 (=
} guiente.

${ }^{1}$ Cf. Aranegui 2004, pp. 194-206, así como la demás bibliografía citada en la nota si- 
clientes y los libertos del anfitrión, una vez borrachos, se enzarzan en una pelea empleando como armas arrojadizas dos elementos de la vajilla: los pocula y las lagonae, indicando mediante la adjetivación Saguntina ... lagona (ablativo instrumental, singular colectivo) que éstas eran de Sagunto. Aquí sí hay una referencia explícita a la antigua industria saguntina, pero -al menos a primera vista y si las palabras en cuestión las entendemos en su sentido propio- no a la del vino sino a la de la alfarería y, en particular, a uno de sus productos: la lagona ${ }^{4}$, recipiente panzudo y de cuello estrecho (análogo a una garrafa o a un cántaro), empleado en las comidas para verter el vino directamente en las copas ${ }^{5}$. La despectiva mención del origen de este utensilio -el adjetivo Saguntina sirve para insistir en la baja calidad de la vajilla puesta a disposición de los clientes, por oposición al lujo de la usada por el anfitriónse inscribe en una tradición epigramática, como evidencian tres epigramas de Marcial en los que la baja calidad de la cerámica saguntina -no bien identificada arqueológicamente ${ }^{6}$ - se nos presenta como proverbial ${ }^{7}$. La interpretación que, de estos versos, acabo de dar es, en mi opinión, la más fácil y coincide en lo esencial con la de los principales comentaristas modernos de Juvenal, quienes, ajenos a la investigación sobre el vino de la antigua Sagunto, no han visto en Saguntina ... lagona más que la mención de un producto de su alfarería ${ }^{8}$, pese a que ya en la Antigüedad hubo quien supuso que había

1898), p. 194; Ferguson 1979, p. 175; Courtney 1980, p. 234; Morton Braund 1996, p. 281 y Santorelli 2013, p. 75 s.

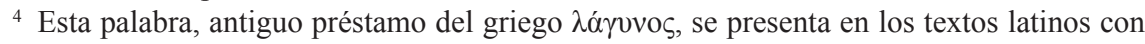
diversas grafías: lagona, laguna, lagoena, lagena, lagyna, lacuna. Cf. ThLL, VII.2, 894, 10 ss., Ernout y Meillet 1985, p. 338, s. u. «lagona».

${ }^{5}$ Cf. Couve, DA 3, 2, 907 s., s. u. «lagena, lagynos»; Pierobon 1979, p. 39; Allison 1999, pp. 63-65; Beltrán, Ortiz y Paz 1999, pp. 169-171.

${ }^{6}$ Cf. Aranegui 2004, pp. 216-221; 1992, p. 38; 2007, pp. 57-59; Arasa 1995, pp. 464-466.

${ }^{7}$ Mart. IV 46.12-16: Piceno quoque uenit a cliente / parcae cistula non capax oliuae, / et crasso figuli polita caelo / septenaria synthesis Sagunti, / Hispanae luteum rotae toreuma; VIII 6.1 s.: Archetypis uetuli nihil est odiosus Aucti / -ficta Saguntino cymbia malo luto; XIV 108 (Calices Saguntini): Quae non sollicitus teneat seruetque minister / sume Saguntino pocula facta luto. Ello parece contradecirse con la noticia de Plin., Nat. XXXV 160: Maior pars hominum terrenis utitur uasis. Samia etiam nunc in esculentis laudantur. Retinent hanc nobilitatem et Arretium in Italia et calicum tantum Surrentum, Hasta, Pollentia, in Hispania Saguntum, in Asia Pergamum.

${ }^{8}$ Mayor $1889^{4}$, p. 248; Friedländer 1895, I p. 262; Ferguson 1979, p. 175; Courtney 1980, p. 234; Morton Braund 1996, p. 282; Santorelli 2013, p. 80. En el mismo sentido, cf. Blümner 1869 , p. 132, así como Schulten, RE I A.2, 1756, 21 ss., s. u. «Saguntum». 
aquí una mención del vino saguntino y de su mala calidad, como un escolio antiguo -que parece remontarse a un comentario de finales del s. IV-atestigua (Schol. in Iuu. V 29, ed. Wessner 1931): ciuitas Spaniae, in cuius territorio malum uinum nascitur ('ciudad de Hispania en cuyo territorio se produce vino malo'). Este escolio, por cuanto sitúa geográficamente la ciudad de Sagunto añadiendo una información sobre la mala calidad de su vino, sugiere que Saguntina ... lagona significa 'garrafa de vino saguntino' y parece suponer que se trata del vino malo referido anteriormente (v. 24 s.). Según creo, esta antigua interpretación es cuestionable (como trataré de demostrar a continuación), y dicha información sobre el vino de Sagunto no necesariamente tuvo que estar basada en un conocimiento previo del escoliasta, sino que pudo ser un mero recurso ideado por él para explicar su suposición de que Juvenal se refiriese aquí, despectivamente, al vino saguntino.

Pero empecemos nuestra discusión reconociendo, como Tovar 1989, p. 287, que estamos ante un caso de posible ambigüedad: «Saguntina ... lagona puede ser interpretado como 'botella (de barro) de Sagunto', o bien como alusión a vino malo» ${ }^{9}$. Para poder entender que Juvenal se refiera aquí al vino de Sagunto, habría que suponer: a) una metonimia del continente por el contenido, por la que lagona estuviera en vez de uinum, como en Mart. IV 69.3 (diceris hac factus caelebs quater esse lagona) y X 45.6 (non facit ad stomachum nostra lagona tuum $)^{10}$, lo que justificaría una interpretación como la de Aranegui 1992, p. 37, cuando parafrasea el pasaje en cuestión en estos términos: «el vino de Sagunto [...] los comensales lo utilizan para lanzárselo de unos a otros en plena diversión» ${ }^{11}$; o b) que el adjetivo Saguntina se refiera, no propiamente a la lagona, sino al vino contenido en ella, de modo que el sintagma Saguntina ... lagona equivaldría a Saguntini (sc. uini) lagona ('garrafa de vino saguntino'), esto es, a una construcción con genitivo de contenido, en la que no habría ninguna ambigüedad (como, p. ej., en Mart. VII 53.6: ... Laletanae nigra lagona sapae). Un uso como éste es el que, por el contexto, hay que reconocer en Mart. XIII 120 (Spoletinum): De Spoletinis quae sunt cariosa lagonis / malueris quam si musta Falerna bibas ${ }^{12}$, VI 89.3 s.: Spoletina data est sed quam siccauerat ipse, / nec fuerat soli tota lagona

9 Cf. Arasa 1995, p. 466.

10 Cf. Fenger 1906, p. 29.

11 Lo mismo se dice en Aranegui 2004, p. 207; 2007, p. 53 s.

12 Cf. comm. ad loc. de Friedländer 1886, II, p. 293 y de Leary 2001, p. 187. 
satis, y X 48.19 s.: de Nomentana uinum sine faece lagona, / quae bis Frontino consule prima fuit.

Paralelos como los que acabo de traer a colación -sobre todo los últimos, en los que el sustantivo lagona está adjetivado por un etnónimo- servirían para demostrar la posibilidad de que en el sintagma Saguntina ... lagona haya una referencia al vino saguntino. Pero pueden hacerse varias objeciones a ello si, además de las posibilidades de la lengua, se considera atentamente tanto el contexto en el que dicho sintagma aparece como la tradición literaria. Un uso metonímico de lagona en vez de uinum es difícil de aceptar aquí, y no ya porque lo lógico sea suponer que en una pelea las «armas» arrojadizas fuesen objetos contundentes (como eran los pocula y las lagonae), sino porque el uso de los diferentes elementos de la vajilla como proyectiles es un tópico en las narraciones de las peleas simposíacas: baste traer a colación, como paralelos más próximos, la narración ovidiana de una pelea simposíaca de la mitología, en la que se enfrentaron los lápitas y los centauros (Ou., Met. XII 235-244), y, sobre todo, la mención que, de esa misma pelea, hay en Mart. VIII 6.7 s. (Hoc cratere ferox commisit proelia Rhoetus / cum Lapithis... (cf. Verg., Georg. II 457) ${ }^{13}$ y de la que Juvenal parece depender directamente en el v. 29 del pasaje en cuestión (pugna Saguntina feruet commissa lagona). Que Saguntina ... lagona signifique 'garrafa de vino saguntino’ parece más probable, pero ello sería incongruente también con la tradición literaria: precisamente con esa tradición epigramática a la que me he referido supra, que está muy bien atestiguada en Marcial -de cuyos epigramas satíricos tanto depende Juvenal- y que consiste en referirse despectivamente a la cerámica (no al vino) de Sagunto ${ }^{14}$. Creo, pues, que es preferible ver un uso propio tanto del sustantivo como del adjetivo en el sintagma Saguntina ... lagona, entendiéndolo como «garrafa saguntina» (esto es, fabricada en Sagunto), del mismo modo que en Mart. IV 46.9 (et nigri Syra defruti lagona «y una garrafa siria de negro vino cocido») el etnónimo Syra se emplea propiamente para indicar que la lagona era una garrafa de vidrio soplado de Siria ${ }^{15}$. Cabría como mucho la posibilidad de que, por ser prover-

\footnotetext{
${ }_{13}$ Más ejemplos de este tópico, en Nisbet y Hubbard 1989 (= 1970), p. 312, comm. ad Hor., Carm. I 27.1.

14 Véanse los textos citados supra nota 7.

15 En este sentido, cf. comm. ad loc. de Moreno 2006, p. 345, así como Stietzel 1907, p. 40; cf. también White 1975, p. 161.
} 
bial -al menos según dicha tradición epigramática y satírica- la baja calidad de la cerámica saguntina, Saguntina ... lagona significara «garrafa como las saguntinas», esto es, «de baja calidad»: de ser así, se trataría de una expresión análoga a Saguntina fames, empleada por Ausonio, Epist. 22.43, para referirse a una hambre extrema (como la que los saguntinos padecieron durante el asedio de su ciudad) ${ }^{16}$.

Bien distinto es el problema que veo en el pasaje de Frontón, 134.17 ss. van den Hout $2^{\mathrm{a}}$ ed., perteneciente a la primera carta (quizá del año 162) del corpus epistolar titulado De eloquentia por Niebuhr y dirigido al emperador Marco Aurelio:

[...] tempus est de uerbis primum quid censeas considerare. Dic sodes hoc mihi: utrumne, tametsi sine ullo labore ac studio meo uerba mihi elegantiora ultro occur $<$ re $>$ rent, spernenda censes ac repudianda an cum labore quidem et studio inuestigare uerba elegantia prohibes, eadem uero, si ultro, si iniussu atque inuocatu meo uenerint, ut Menelaum ad epulas quidem, recipi iubes? Nam istud quidem uetare durum prosus atque inhumanum est: consimile ut si ab hospite, qui te Falerno accipiat quod rure eius natum domi superfiat, Cretense postules uel Saguntinum, quod malum foris quaerendum sibi atque mercandum sit.

[...] es hora de examinar, en primer lugar, cuál es tu opinión acerca del vocabulario. Dime, por favor, lo siguiente: pese a que sin ningún esfuerzo ni empeño por mi parte se me ocurrieran espontáneamente palabras algo elegantes, ¿opinas que debería despreciarlas y rechazarlas? ¿O es que, por cierto, me prohíbes rastrear con esfuerzo y empeño palabras elegantes pero, al mismo tiempo, me autorizas a recibirlas en el caso de que me lleguen espontáneamente, precisamente como Menelao al banquete, sin autorización ni invitación mías? La verdad es que prohibir eso es absolutamente duro e inhumano: casi lo mismo que si a un huésped que te acogiera con vino de Falerno, que por ser producto de su finca hubiera de sobra en su casa, le pidieras un vino cretense o un saguntino, que, siendo malo, tuviera él que ir a buscar y comprar fuera de casa.

Frente a la puntuación de van den Hout y de los demás editores al final de este pasaje (... quod, malum, foris...), basada en la suposición de que malum esté empleado como interjección de maldición o indignación ('¡idiablos!', '¡maldita sea!’), he quitado las comas porque prefiero entender, como Hof-

${ }^{16}$ Cf. Otto 1890, p. 305. 
mann $1985^{2}$, p. 360, que está empleado como adjetivo, con el que Frontón subraya la mala calidad de los dos vinos extranjeros mencionados por oposición al buen vino de Falerno: el uso de malum como interjección se da siempre después de un pronombre interrogativo o exclamativo (cf. ThLL, VIII 236, 56 ss.), nunca después de un relativo, que es lo que aquí hay; obsérvese, además, que -según el análisis sintáctico y la puntuación que defiendo- estamos ante la construcción consistente en la inclusión dentro de la proposición de relativo de un adjetivo en función predicativa y referido al antecedente (cf. Kühner y Stegmann 1988, II, p. 311 s.): Cretense ... uel Saguntinum, quod malum ..., en paralelo con la secuencia inmediatamente precedente: Falerno ... quod ... natum ...

Pero, en cuanto al asunto que ahora nos ocupa, veo en este pasaje un problema gráfico-fonético y de crítica textual, por el que puede cuestionarse su supuesta validez como testimonio del vino saguntino. Me parece bastante probable que el segundo de esos dos vinos extranjeros mencionados por Frontón sea, no el de Sagunto, sino el de la isla griega de Zacinto, como ya Orelli 1830, p. 170 -en una de sus emendationes al texto frontoniano, que no ha sido aceptada por ningún editor posterior- observó: «Vinum Saguntinum intellige Zacynthium; nisi sic scribendum», lo que quiere decir que se trata, no del vino de Sagunto, sino del de Zacinto, ya sea porque Frontón empleara Saguntinus, -a, -um como etnónimo de Zacinto, ya sea porque la tradición manuscrita hubiese alterado la forma Zacynthium transformándola en Saguntinum. La primera explicación podría aceptarse si se admite que -como yo mismo traté de demostrar ${ }^{17}$ a propósito de Cato, Agr. 8.1, donde ficos Sacontinas parece equivaler a ficos Zacynthias «higueras zacintias»: cf. Plaut., Merc. 943: Hospes respondit Zacynthi ficos fieri non malas- el etnónimo de Zacinto podía presentarse, al menos en el latín arcaico, bajo dos formas distintas: Sacuntinus (o Sacontinus, con el vocalismo que aparece en los códices del texto de Catón) y Sacuntius (escrito Zacynthius desde la época clásica); lo que concuerda con una noticia de Prisciano (Gramm. Lat. Keil II 36, 20), según la cual los ueteres escribían Saguntum (yo preferiría la variante Sacuntum, lección de dos de los códices colacionados por Keil) para transcribir Zókvv $\theta o \varsigma^{18}$. De ser así, es posible que Frontón, conforme a su tendencia ar-

\footnotetext{
17 Hernández 2011.

${ }^{18}$ Cf. Lindsay 1894, p. 104. Añádase esta noticia de Prisciano a los datos recopilados y analizados por Santiago 1990 en su estudio sobre los nombres antiguos de Sagunto.
} 
caizante, recuperase la forma arcaica Sacuntinus -que quizá siguiese viva en la lengua popular-pero con una $g$ en vez de $c$ (Saguntinus), que pudo estar motivada (si es que no estamos ante una confusión de la tradición manuscrita) por la misma razón -pero en sentido inverso- por la que, en vez de Saguntum, se emplearon formas artificiosas y helenizantes como Sacynto (ablativo) (en la marca anfórica de $C I L$ II $6254_{9}$ y $C I L$ XV 2632, a la que me he referido supra), Zacynthos (Iuu. XV 114) o Saguntos (Sil. I 502 y 573, II 446, III 178, IX 186; Flor., Epit. I 22.3): para subrayar la supuesta vinculación de Sagunto con Zacinto, la que -según la leyenda- había sido su metrópolis. Lo mismo cabe decir de la forma empleada por Salustio, Hist. fr. II 65 Maurenbrecher (apud Char., Gramm Lat. Keil I 143, 9 s.), como etnónimo correspondiente a Saguntum: Saguntius, - $a$, -um, resultante, según parece, de la

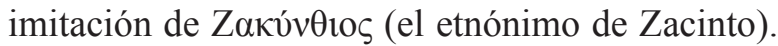

Ahora bien, quizá la otra explicación posible, que es precisamente la menos complicada, sea más probable: esto es, que se trate de un error de transmisión, habida cuenta que los topónimos Saguntum y Zacynthos (-us), así como sus etnónimos respectivos, Saguntinus y Zacynthius, se confunden a menudo en la tradición manuscrita: cf., p. ej., las lecciones Sagunto en vez de Zacyntho y Saguntumque en vez de Zacynthumque en Liu. XXI 7.2 y XXXVI 42.5 respectivamente, así como Scrib. Larg. 208, donde la tradición manuscrita da la lección bituminis Saguntini, quod est natura liquidum, que en la edición de Sconocchia 1983 se corrige leyendo Zacynthii y no Saguntini, aceptando la conjetura de Rhodius 1655, p. 294 («lege omnino Zacynthii»), basada en testimonios como Plin., Nat. XXXV 178 (est uero liquidum bitumen, sicut Zacynthium et quod a Babylone inuehitur) y Gal. XIII

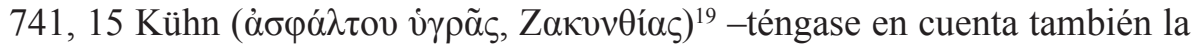
lección Zacontini de dos testimonios de tradición indirecta, que es análoga a Sacontinas (= Zacynthias) de Cato, Agr. 8.1 (cit. supra) y que, según Sconocchia 1995, p. 312 s., «sembra potersi spiegare come una fase intermedia del processo di corruzione che ha portato alla "normalizzazione" Saguntini». Tales confusiones se debieron a la gran semejanza fónica existente entre ambos topónimos y entre sus respectivos etnónimos: por el proceso llamado

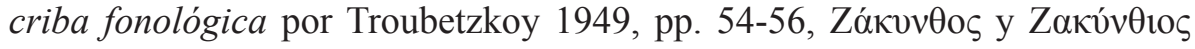
(transcritos en latín clásico como Zacynthos (-us) y Zacynthius) sonaban respectivamente Sacuntos (-us) y Sacuntius en boca de los hablantes latinos,

${ }^{19}$ Cf. FHA, VIII, p. 405; Schulten 1955-57, I, p. 459. 
y así es como tenían que escribirse en el latín arcaico ${ }^{20}$, antes de que empezaran a utilizarse los dígrafos $\mathrm{PH}, \mathrm{TH}$ y $\mathrm{CH}$ y las letras $\mathrm{Y}$ y Z $\mathrm{Z}^{21}$. También el mito de la fundación de Sagunto por colonos procedentes de Zacinto, basado precisamente en la semejanza existente entre ambos topónimos, debió de contribuir a que se produjeran dichas confusiones.

Hay, además, en el contexto un argumento que, añadido a los que acabo de aducir, demostraría que la posibilidad de que Saguntinum esté en vez de -o equivalga a- Zacynthium es mucho mayor. Tengamos en cuenta que Frontón expone aquí su posición con respecto al delectus verborum o 'selección del vocabulario' -lo que fue su principal preocupación retórica- concluyendo con un símil que debe entenderse como una defensa del uso de palabras genuinamente latinas frente a la importación de palabras griegas ${ }^{22}$ : por oposición al vino de Falerno, que simboliza el rico y buen patrimonio léxico del latín, los dos vinos malos y de importación mencionados a continuación representan las palabra extranjeras, que -habida cuenta de cuál es la polémica planteada y de que se trata de la lengua literaria latina, en la que no cabría la consideración de la influencia de una lengua extranjera que no fuese el griego- deben entenderse, en particular, como palabras griegas. Es por ello por lo que en un símil como éste resulta más adecuado, en mi opinión, oponer al buen vino de Falerno - vino de Italia y, por tanto, nacionaldos malos vinos griegos, el de Creta y el de Zacinto, que uno griego (de Creta) y otro de Hispania (de Sagunto). Además, la baja calidad de ambos vinos griegos ${ }^{23}$ está bien atestiguada en la literatura antigua: el de Creta era un vino de pasas (passum) poco apreciado en Roma, a juzgar por lo que de él dice Marcial, XIII 106.2: ... mulsum pauperis esse solet («suele ser el vino mulso del pobre») $)^{24}$; y el vino de Zacinto -el que ahora nos interesa- sabemos que era considerado malsano por la noticia de Ateneo, I 33b Kaibel: ó

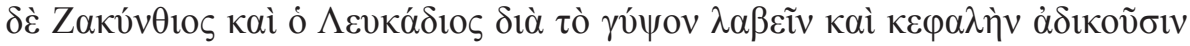
(«el vino de Zacinto y el de Léucade, por contener yeso, también hacen daño a la cabeza» $)^{25}$.

${ }^{20}$ Cf. Moralejo 2011, p. 295; Hernández 2011, p. 191.

${ }^{21}$ Cf. Traina $2002^{5}$, pp. 17-19.

22 En este sentido, cf. Brock 1911, pp. 38-41; Portalupi 1961, pp. 115-118 y Valette 2013, p. 116 s., así como la conversación del propio Frontón apud Gell. II 26 (fr. 5 van den Hout).

${ }^{23}$ Cf. Jardé, $D A$ 5, 914 s., s. u. «vinum».

${ }^{24}$ Cf. van den Hout 1999, p. 322.

${ }^{25}$ Cf. Jacob, $D A$ 2.2, 1715, s. u. «gypsum». 
En conclusión: es muy probable que ninguno de los dos pasajes examinados se refiera al vino saguntino. El pasaje de Juvenal parece referirse, más bien, a la cerámica saguntina, considerada como de baja calidad de acuerdo con la tradición epigramática y satírica. El pasaje de Frontón, por su parte, se referiría, en realidad, al vino de Zacinto y sería, pues, un ejemplo más de la frecuente confusión, en la tradición manuscrita, de Saguntum y Zacynthos (-us) y de Saguntinus y Zacynthius, de modo que tendría, incluso, que dejar de ser considerado como fuente de la historia antigua de España.

\section{BibLIOGRAFÍA}

Allison, P. M. 1999: «Labels for ladles: Interpreting the material culture of Roman households», en Allison, P. M. (ed.) The Archaeology of Household Activities, Londres - Nueva York, pp. 57-77.

Aranegui Gascó, C. 1990: «Arqueología del vino. Sagunto», Braçal 3, pp. 11-16.

Aranegui Gascó, C. 1991: «El vino de Saguntum», Saguntum y el mar, Valencia, pp. 49-52.

Aranegui Gascó, C. 1992: «Testimonios del vino saguntino, entre otras cuestiones», en Miscel-lània arqueologica a J. M. Recasens, Tarragona, pp. 35-43.

Aranegui Gascó, C. 1999: «El comercio del vino en la costa mediterránea española en época romana», en Celestino Pérez, S. (ed.), El vino en la Antigüedad romana, Madrid, pp. 79-96.

Aranegui Gascó, C. 2004: Sagunto. Oppidum, emporio y municipio romano, Barcelona.

Aranegui Gascó, C. 2007: «Sagunt i Roma», Braçal 35-36, pp. 13-64.

Arasa Gil, F. 1995: Territori i poblament en època romana a les comarques septentrionals del litoral valencià, tesis doctoral, Valencia.

Beltrán Lloris, M, Ortiz Palomar, Ma E. y Paz Peralta, J. A. 1999: «La vajilla relacionada con el vino en Hispania», en Celestino Pérez, S. (ed.), El vino en la Antigüedad romana, Madrid, pp. 129-200.

Blümner, H. 1869: Die gewerbliche Thätigkeit der Völker des klassischen Alterthums, Leipzig.

Brock, M. D. 1911: Studies in Fronto ad his Age, Cambridge.

CIL: Corpus Inscriptionum Latinarum, Berlín, 1863 ss.

Cisneros Fraile, F. 2002: «El vino en el hinterland del Portus de Saguntum (ss. I a. C. - I d. C.)», Saguntum 34, pp. 127-135.

Courtney, E. 1980: A Commentary on the Satires of Juvenal, Londres.

DA: Daremberg, G., Saglio, E. y Pottier, E. (eds.), Dictionnaire des antiquités grecques et romaines, Paris 1877-1919. 
Duff, J. D. 1957 (= 1898): D. Iunii Iuvenalis Saturae XIV, Cambridge.

Ernout, A. y Meillet, A. $1985\left(=1959^{4}\right)$ : Dictionnaire étymologique de la langue latine. Histoire des mots, París.

Fenger, R. 1906: De metonymiae in epigrammatis Martialis usu, Jena.

Ferguson, J. 1979: Juvenal. The Satires, Londres.

FHA: Grosse, R., Fontes Hispaniae antiquae, VIII, Las fuentes desde César hasta el siglo $V d$. de J. C., Barcelona 1959.

Friedländer, L. 1886: M. Valeri Martialis epigrammata libri, Leipzig.

Friedländer, L. 1895: D. Iunii Iuvenalis Saturarum libri V, Leipzig.

Hernández Pérez, R. 2011: «Ficos Sacontinas (Cato, Agr. 8.1): de Zacinto, no de Sagunto», Emerita 79, pp. 189-194.

Hofmann, J. B. 1985²: La lingua d'uso latina, trad. L. Riccottilli, Bolonia.

Kühner, R. y Stegmann, C. 1988 (=19765): Ausführliche Grammatik der lateinischen Sprache. II: Satzlehre, I-II, Darmstadt.

Leary, T. J. 2001: Martial Book XIII: The Xenia, Londres.

Lindsay, W. M. 1894: The Latin Language. An Historical Account of Latin Sounds, Stems, and Flexion, Oxford.

Mayor, J. E. B. 18894: Thirteen Satires of Juvenal, Londres.

Moralejo, J. L. 2011: «Mumio vuelve a Itálica (de nuevo sobre CIL I² 630, II 1119)», ZPE 177, pp. 289-297.

Moreno Soldevila, R. 2006: Martial, Book IV: a Commentary, Leiden.

Morton Braund, S. 1996: Juvenal, Satires. Book I, Cambridge.

Nisbet, R. G. M. y Hubbard, M. 1989 (= 1970): A Commentary on Horace: Odes, Book I, Oxford.

Orelli, G. C. 1830: C. Cornelii Taciti Dialogus de oratoribus ... accedunt... et Chrestomathia Frontoniana..., Zúrich.

Otto, A. 1890: Die Sprichwörter und sprichwörtlichen Redensarten der Römer, Leipzig.

Pierobon, R. 1979: «Lagynos: funzione e forma», Rivista di studi liguri 45, pp. 27-50.

Portalupi, F. 1961: Marco Cornelio Frontone, Turín.

RE: Pauly, A. y Wissowa, G. (eds.), Real Encyclopädie der klassischen Altertumswissenschaft, Stuttgart 1894 ss.

Rhodius, I. 1655: Scribonii Largi Compositiones medicae, Padua.

Santiago, R. A. 1990: «En torno a los nombres antiguos de Sagunto», Saguntum 23, pp. 123-140.

Santorelli, B. 2013: Giovenale, Satira V. Introduzione, traduzione e commento, Berlín - Boston.

Schulten, A. 1955-57: Iberische Landeskunde. Geographie des antiken Spanien, Estrasburgo. 
Sconocchia, S. 1983: Scribonii Largi Compositiones, Leipzig.

Sconocchia, S. 1995: «Nuovi testimoni scriboniani tra tardo antico e medioevo», RFIC 123, pp. 278-319.

Stietzel, W. 1907: De synecdocha eiusque in Martialis epigrammatis usu, Jena.

THA: Mangas, J. y Myro, $\mathrm{M}^{\mathrm{a}}$ del Mar (eds.), Testimonia Hispaniae antiqua. III: Medio físico y recursos naturales de la Península Ibérica en la Antigüedad, Madrid 2003.

ThLL: Thesaurus linguae Latinae, Leipzig 1900 ss.

Tovar, A. 1989: Iberische Landeskunde II. Las tribus y las ciudades de la antigua Hispania, 3: Tarraconensis, Baden-Baden.

Traina, A. 20025: L'alfabeto e la pronunzia del latino, Bolonia.

Troubetzkoy, N. S. 1949: Principes de phonologie, trad. J. Cantineau, París.

Valette, E. 2013: «Le vetêment bigarré des danseurs de Pyrrhique: pratiques du bilinguisme dans la correspondance de Fronton et Marc Aurèle», en Mestre, F. y Gómez, P. (eds.), Three Centuries of Greek Culture under the Roman Empire. Homo Romanus Graeca oration, Barcelona, pp. 101-123.

van den Hout, M. P. J. 1988²: M. Cornelii Frontonis epistulae, Leipzig.

van den Hout, M. P. J. 1999: A Commentary on the Letters of M. Cornelius Fronto, Leiden.

Wessner, P. 1931: Scholia in Iuvenalem vetustiora, Leipzig.

White, K. D. 1975: Farm Equipment of the Roman World, Cambridge.

Fecha de recepción de la primera versión del artículo: 24/05/2016

Fecha de aceptación: 01/09/2016

Fecha de recepción de la versión definitiva: 21/09/2016 


\section{NOTAS E INFORMACIÓN}

\section{Nota crítica a dos lugares oscuros de Ovidio (Met. XIII 884 y 890 )*}

A critical note on two problematic passages of Ovid (Met. XIII 884 and 890)

\section{Luis Rivero García}

Universidad de Huelva

lrivero@uhu.es

ORCID iD: http://orcid.org/0000-0001-7140-2072

Se analizan dos pasajes de las Metamorfosis de Ovidio y se sugieren propuestas de enmienda.

Palabras clave: Ovidio; Metamorfosis; crítica textual.

Two passages of Ovid's Metamorphoses are considered, and an attempt is made to correct them.

Key words: Ovid; Metamorphoses; textual criticism.

Cómo citar este artículo / Citation: Rivero García, Luis 2017: «Nota crítica a dos lugares oscuros de Ovidio (Met. XIII 884 y 890)», Emerita 85 (1), pp. 153-159.

Ou., Met. XIII 882-892:

Insequitur Cyclops partemque e monte reuulsam

mittit, et extremus quamuis peruenit ad illum

angulus †e saxo†, totum tamen obruit Acin.

At nos - quod fieri solum per fata licebat -

* El presente artículo, elaborado en los primeros meses de 2015 en la Academia Americana de Roma y en la Biblioteca Vaticana gracias a una beca del Ministerio de Educación, se inserta en el Proyecto de Investigación FFI2013-42529, financiado por el MICINN, Gobierno de España. El texto y aparato crítico, así como la propia argumentación pertenecen al comentario crítico textual que actualmente preparo sobre el libro XIII de las Metamorfosis. Los manuscritos, agrupados por bloques cronológicos, así como las ediciones se citan de forma abreviada, siguiendo las propuestas disponibles en http://www.uhu.es/proyectovidio/ esp/index.html. El texto de Planudes se cita por la edición de Papathomopoulos-Tsavare 2002. 
fecimus ut uires adsumeret Acis auitas.

Puniceus de mole cruor manabat, et intra temporis exiguum rubor euanescere coepit fitque color primo turbati fluminis imbre purgaturque mora. Tum moles iacta dehiscit uiuaque per rimas proceraque surgit harundo osque cauum saxi sonat exsultantibus undis

- $\underline{84}$ e saxo $\Omega$, edd.; uix sanum : ex saxo V2 B4Ba (p.c.)CDeDrFGgO3V5V9(p.c. a m.p.) Cs2Li3P8 O10²(i.l. p.c.) P41, prob. Ellis, Edwards 1905 : is motus M(p.c., -us i.ras.; quid a.c. n.l.) Lr27 : asaxo AbMoV9(a.c.) Ft : a saxo Bs4 Es4 : exaxo B3 : exasto $B a($ a.c.) : est saxo Lr8 O10 Es6 : quid $N$ a.c. (e saxo i.ras.) n.l. : is montis Merkel 1850 : is molis Merkel 1875, Zingerle 1884, Polle 1888, H-K-E 1898, Keene 1898, Magnus 1914, Ehwald 1915, van Proosdij 1951, H-K-E-A 1966, Fink 2007 : hic iactu Riese 1872(is i. dub. in notis) : ex iactu Riese 1889 : exiguus Hellmuth - $\underline{890}$ iacta B2Ld Sp Rd, Plan., malit Glareanus, prob. Heinsius 1659(qui et «in ... tribus» test.), Burm. 1727, Walch. 1731, Jahn 1832, Weise 1845, Koch 1866, H-K-E 1898, Goold 1984 : tacta $\Omega$, Puteol. 1471, Venet. 1472, Calph. 1474, Accurs. 1475, Regius 1493, Aldina 1502, Naug. 1516, Ciof. 1575, Heinsius 1652, Magnus 1914, Ehwald 1915(iacta H-K-E 1898), Lafaye 1930, Trepat-de Saav. 1932, van Proosdij 1951, Breitenbach 1964, H-K-E-A 1966, Anderson 1982, Segura 1983, Hill 2000, Hopkinson 2000, R. de Verger 2005, Tarrant 2004(qui tamen suspectum hab.), dub. Lemaire 1822(in notis) : tales Li3 : fracta $A L u A 4^{3}$ (mg.)Ba(p.c.)DeFe (mg. u.l.) $G^{3}(m g .$, add. «i. aperta»)L4(tacta a.c.)LsN2TrVd(p.c., fort. a m.p.) B8Ds2 (i.l. u.l.) P10So BoBo2Es22(p.c.)P28(p.c.) B14Es5 Mv7, "prim. Moret. et quindecim alii» test. Heinsius 1659, prob. Gierig 1807, Bothe 18182, Lemaire 1822, Richter 1828, Baumg.-Crus. 1834, Bach 1836, Loers 1843, Merkel 1850, Riese 1872, Korn 1880, Zingerle 1884, Polle 1888, Simmons 1889, Edwards 1905, Fabbri 1923, Fink 2007, dub. Tarrant 2004(in app.) : facta $G^{2}$ (p.c.; tacta G) Ba(a.c.) : fata $E s^{3}:$ tracta FO3(per comp.)V4²(r suprascr.)V5(tacta p.c.) O10(tacta p.c.)P28(a.c.), «in utroque meo, Urs. Farnes. et cod. Vatican.» test. Ciof. 1575, dub. Ellis : tecta Lr3(tacta p.c.) Lr22V30, Aler. 1471, Lugd. 1546, Lugd. 1565, Glar-Long. 1570, Bersm. 1596 : tanta A3 To(tacta $m g$. u.l. $\left.T o^{2}\right)$ : tota $A b\left(\operatorname{tacta}\right.$ i.l. u.l. $\left.A b^{2}\right)$, coni. Hardie : acta Lr4Lr6(uid.) : capta $V d($ a.c.) : rupta $P 41$ : cuncta $B s 4$ (tracta uel tincta i.l. u.l.) : certa «in nonnullis» test. Heinsius 1659 : taetra Merkel 1875 : torta fort. legendum •

En el v. 884, perteneciente al episodio de la muerte de Acis a cargo de Polifemo, el ms. $M$ (s. $\left.\mathrm{XI}^{2}\right)$ nos deja la extraña lectura is motus ( $M^{2}$ sobreescribe «s. angulus»), en la que $-u s$ está escrito sobre algo borrado y hoy ilegible; $V 2\left(\mathrm{~s} . \mathrm{XI}^{2}\right)$ y un grupo de recentiores dan ex saxo, grafía que recibió la aprobación de R. Ellis al hallarla sobreescrita como corrección de est saxo en $\mathrm{O}^{2} 0^{2}$ (s. XIV ${ }^{\mathrm{ex}}$ ), sobre el muy discutible argumento de que «est, proves ex not $e$ to be right» (ap. Simmons 1889, p. 167), y 
esta variante fue llevada a su texto por Edwards (1905, p. 479; para ex saxo cf. e.g. Vitr., VIII 6.8, pero para e saxo cf. e.g. Cato, Orig. II 16); en $N$ (s. XI ${ }^{\text {ex }}-\mathrm{XII}{ }^{\mathrm{in}}$ ) la lectura e saxo está escrita sobre algo borrado que tal vez podría haber sido is motus pero que hoy resulta imposible de determinar con una mínima fiabilidad.

En su $1^{\mathrm{a}}$ edición (1850) Merkel subió al texto su conjetura is montis (cf. 882), no secundada por ningún otro editor, pero en la $2^{\mathrm{a}}$ editó is molis a partir de $887 \mathrm{y}$ 890 (cf. VIII 357: véase Merkel 1875, p. XXXVIII), retrotrayendo la coma a illum (v. 883), conjetura que sí ha sido seguida por Ehwald, Magnus y otros editores hasta nuestros días. Riese, por su parte, editó hic iactu en su $1^{\mathrm{a}}$ edición, aunque dejando abierta en sus notas la posibilidad de leer alternativamente is iactu (1872, p. XXIV). En su $2^{a}$ edición cambió de idea y sostuvo $(1889$, p. XXVI) que la parádosis e saxo era corrupción de ex iactu a través de una grafía facxo. Por último, Hellmuth (1880, pp. 28-29) comienza señalando lo inaceptable de la expresión angulus e saxo en lugar de angulus saxi y a continuación aborda las limitaciones de las propuestas de Merkel y de Riese, sobre todo en lo relativo a los pronombres mostrativos y fóricos, para a continuación proponer la interesante conjetura angulus exiguus, estilísticamente lograda por la yuxtaposición significativa exiguus totum, que él apoya en paralelos como VIII 337: longa paruae, XI 506: inferno summum, o XII 495: in hunc omnes unum. Además, el adjetivo podría evocar el modelo virgiliano (Aen. X 128): haud partem exiguam montis. Por lo que respecta a la doble adjetivación de angulus, Hellmuth recuerda que ésta no es obstáculo cuando uno de los adjetivos tiene valor local, para lo que compara XI 197-198: dextera Sigei, Rhoetei laeua profundi / ara Panomphaeo uetus est sacrata Tonanti, y XV 443: externum patria contingat amicius aruum. Desde el punto de vista paleográfico la conjetura es creíble respecto de e saxo, aunque Hellmuth, tratando de dar cuenta de la lectura de $M$, explica la corrupción a partir de una repetición de extremus, del verso anterior, lo que habría forzado a los copistas a rehacer el texto, siendo el copista de $M$ el más «audaz» en su reconstrucción, pero esto no deja de ser una forma de reconocer que no se sabe cómo pudo escribir aquel copista lo que escribió. La propuesta de Hellmuth, más fina que las de Merkel y Riese, tiene sin embargo el inconveniente de ser redundante, pues la expresión extremus angulus tiene ya la precisa finalidad de indicar que lo que cayó sobre Acis fue una parte exigua - un borde, un pico- de la porción de monte arrancada por Polifemo.

Aun así, sigue siendo evidente que e saxo no va bien porque, como acertadamente señalaba Hellmuth, aquí tendría que equivaler a un simple genitivo («ungewöhnliche adnominale partitive e», según Bömer 1982, p. 448, sin más argumentación), y eso no es aceptable en el latín de Ovidio (y tampoco tiene sentido como separativo ni dependiendo de peruenit ni de angulus), pero además porque e saxo es una expresión rara y limitada a la prosa con la sola excepción de este pasaje y porque aparece sospechosamente cerca de e monte (v. 882). 
El sintagma e saxo podría ser deformación gráfica de la lectura genuina, pero también podría proceder de una glosa a un participio concertado con angulus del tipo excisus, exceptus, excussus o exsectus, i. e. «una punta marginal desgajada (de la roca)». Del mismo modo, is motus podría ser glosa de un participio, pero en este caso concertado con el sujeto de obruit, como eiectus (aunque con el sentido de 'arrojar violentamente' Ovidio prefiere eiectare: cf. II 231, V 353), exactus (véase Bömer 1976, pp. 214 y 265, coment. a IV 734 y V 171), excissus (aquí con el sentido de 'arrojado': cf. e.g. Tac., Hist. IV 23.3), exceptus (aquí, 'recibido') o inmissus.

La lectura de $M$ (y tal vez -recuérdese- de $N$ a.c.) también podría ser deformación gráfica o bien glosa de un participio del tipo euoluens (cf. XII 519), inuolitans, intortus, o de una locución como in gyrum/-os, i.e. «aunque llega hasta él el extremo del borde girando» (cf. e.g. Verg., Aen. VII 378-381), que habría podido venir sugerido a Ovidio por é $\pi \imath \delta \imath v \dot{\sigma} \sigma \alpha \varsigma$ ('haciéndola girar': véase Heubeck 1992, p. 41) en el

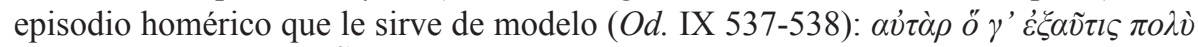

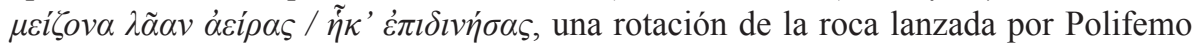
que se corresponde al conocido movimiento de taladro con el que Odiseo y los suyos le habían hundido la estaca en el ojo (Od. IX 383-386, con Heubeck 1992, p. 34, coment. a 382-390; E., Cyc. 460-463; Verg., Aen. III 635). No encuentro, sin embargo, ningún pasaje dentro o fuera de la obra de Ovidio que avale con suficiente nitidez ninguna de estas propuestas, por lo que de momento me limito a señalar como corrupto el texto transmitido.

El segundo pasaje objeto de discusión está en el v. 890 y puede tener, como veremos, alguna relación con el anterior. La lectura ampliamente mayoritaria de los manuscritos es moles tacta, que también ha sido adoptada por una mayoría de editores (uid. app.), tanto entre las ediciones más antiguas como por parte de Heinsius en su $1^{\mathrm{a}}$ edición (1652), y muy especialmente desde que Magnus la recuperara con el discutible argumento de que se trata de un «toque mágico» $(1914$, p. 524: «a me sc, cf 652, 886, XI 308»; véase además Bömer 1982, p. 449), lo que en todo caso habría necesitado de un agente más explícito (véase Hill 2000, p. 168; Hopkinson 2000, p. 230; Hardie 2015, p. 358; todos con dudas respecto de la lectura). El último editor que la ha mantenido ha sido Tarrant, quien sin embargo muestra (2004, p. 406 in app.) sus dudas sobre ella y sus simpatías por fracta («fort. recte»; una posición inversa vemos en Lemaire 1822). Copistas y editores han sentido la necesidad de glosar el participio, lo que es indicio de que no se encuentran del todo cómodos con él. Así, por ejemplo, en $N^{2}$ encontramos sobreescrito unda, en $M 2^{2}$ vemos a sanguine (sanguine en $\mathrm{V}^{2}$ ) y en $\mathrm{Fe}^{2}$ leemos ab aqua. Regius, por su parte, no especifica el origen del contacto (1493, ad loc.): «Tum rupes percussa aperitur».

Otra de las variantes mejor atestiguadas es fracta, que no fue llevada a su texto pero sí recibió el beneplácito («bene») de Heinsius (1659, pp. 354-355) y que fue editada por Gierig $(1807$, p. 303$)$ por comparación con XII 488: fractaque dissiluit 
percusso lammina callo, y con Verg., G. IV 410: in aquas tenues dilapsus abibit, pasajes ambos de muy escaso valor probatorio (cf. Luck 2009, p. 113). Esta lectura ha sido aceptada por algunos editores hasta nuestros días. Bach $(1836$, p. 362) cree que de ella surgió tracta y de ésta a su vez tacta. El problema es que fracta dehiscit es una redundancia que delata el origen espurio de fracta (cf. rupta en el ms. P41).

Otra variante interesante, la de grafía más cercana a tacta, es iacta, que he encontrado tan sólo en $B 2 L d S p R d$ y que debió de encontrar Planudes en su antígrafo, pues tradujo $\beta \lambda \eta \theta \varepsilon \tilde{\varepsilon} \sigma \alpha$. Esta lectura recibió la aprobación de Glareanus (Glar.-Long. 1570, p. 228: «Omnes tacta legunt. At ego iacta malim. Intelligo enim saxum a Polyphemo iactum»; cf. Bersm. 1596, p. 547) y fue llevada a su texto por Heinsius en su $2^{\text {a }}$ edición a partir de la paráfrasis «iactis in altum molibus» $(1659$, p. 355), paráfrasis desde luego innecesaria frente a la más simple de Glareanus. Entre los editores recientes tan sólo Goold (1984; así ya Miller 1921²) la ha aceptado.

Mucho menos creíble resulta la variante tracta, pues la piedra fue arrojada, como se dice en el v. 883 (mittit), y no arrastrada (compárese además la variante acta). Ciofanus $(1575$, p. 187) la atestigua en varios de sus manuscritos y esta variante recibió la tímida aprobación de Ellis, quien la había encontrado de nuevo en 010 (ap. Simmons 1889 , p. 168). Otras variantes transmitidas son tecta, tanta, facta, capta, tincta, cuncta o certa. Semánticamente cercana a tanta y, más aún, a cuncta está la variante tota, que he encontrado en $A 3$ y en To y que Tarrant (2004, p. 406) recogía como propuesta de Hardie (el propio Hardie 2015, p. 72 la recoge in app., aunque no la lleva al texto ni la menciona en su comentario: p. 358). Si paleográficamente es viable, tiene además a su favor la yuxtaposición significativa tota dehiscit. Muy desafortunada, en cambio, fue la propuesta taetra de Merkel para su $2^{\mathrm{a}}$ edición (1875; en 1850 había editado fracta).

La parádosis tacta tal vez nos permitiría sugerir torta (i. e. torta $>$ tarta $>$ tacta), pues la roca fue arrojada (cf. IV 709-710: quantum Balearica torto / funda potest plumbo medii transmittere caeli; epist. IV 158: ueniant proaui fulmina torta manu), quizá incluso con efecto giratorio, tal como apuntábamos más arriba en nuestra discusión sobre e saxo. Ante las limitaciones de tacta me inclino entre dudas por iacta como variante más probable tanto por el significado como por la grafía, que habría dado lugar a tacta y a las demás a partir de ésta.

\section{BiBLIOGRAFÍA}

Bach, E. C. Chr. 1836: P. Ovidii Nasonis Metamorphoseon libri XV, mit kritischen und erläuternden Anmerkungen von ... Vol. II, Hannover.

Bersman, G. 1596: Publ. Ovidii Nasonis Operum Tomus II. quo continentur Metamorphoseon libri XV. Ex postrema Iacobi Micylli recognitione, et recensione nova Gregorii Bersmani ..., editio tertia aliquot locis auctior, Leipzig. 
Bömer, F. 1976: P. Ovidius Naso. Metamorphosen. Kommentar von ... Buch IV-V, Heidelberg. Bömer, F. 1982: P. Ovidius Naso. Metamorphosen. Kommentar von ... Buch XII-XIII, Heidelberg.

Ciofanus, H. 1575: Herculis Ciofani, Sulmonensis, in P. Ovidii Metamorphoseon ex XVII antiquis libris Observationes ..., Venecia.

Edwards, G. M. 1905: Corpus poetarum Latinorum a se aliisque denuo recognitorum et brevi lectionum varietate instructorum edidit I. P. Postgate. Tom. I. Editio altera emendatior (= 1894). Metamorphoseon libri XV, recogniti a G. M. Edwards, Londres.

Ehwald, R. 1915: P. Ovidius Naso. Opera. Vol. II, Metamorphoses, editio maior, Leipzig.

Gierig, G. E. 1807: P. Ovidii Nasonis Metamorphoses. Recensuit varietate lectionis notisque instruxit et indices duos, unum verborum, alterum nominum propriorum adiecit Gottlieb Erdmann Gierig. Editio altera ad singulos prope versus vel emendatior vel auctior. Tomus posterior [Met. IX-XV], Leipzig.

Glareanus, H. 1570: P. Ovidii Nasonis opera, veterum exemplarium auxilio ab infinitis mendis emendata. Henrici Glareani annotationes in Metamorphosin \& ad verba \& ad res intelligendas magni usus. Praeterea Longolij, quae lectorem plurimum in impeditis locis iuuare possunt. Item, fragmenta quaedam Ouidij ex libris, qui magna parte periere, epigrammaton $\&$ non male natum carmen ad Pisonem. Excudebat Ioannes Kyngstonus (s.l.).

Goold, G. P. 1984: Ovidius. Metamorphoses. With an English Translation by F. J. Miller, Revised by G. P. Goold, Londres - Cambridge (Mass.).

Hardie, Ph. 2015: Ovidio, Metamorfosi, volume VI (libri XIII-XV), a cura di Ph. Hardie, testo critico basato sull'ed. oxoniense di R. Tarrant, trad. di G. Chiarini, Milán.

Heinsius, N. 1652: Operum P. Ovidii Nasonis editio nova, accurante Nicolao Heinsio, Dan. fil., II, Amsterdam.

Heinsius, N. 1659: Operum P. Ovidii Nasonis editio nova. Nic. Heinsius, Dan. F. recensuit, et notas addidit, II, Amsterdam.

Hellmuth, C. 1880: Emendationsversuche zu Ovids Metamorphosen, Progr. Caesareolutreanum.

Heubeck, A. 1992: A Commentary on Homer's Odyssey, vol. II (books IX-XVI), Oxford.

Hill, D. E. 2000: Ovid. Metamorphoses XIII-XV and indexes, ed. with an introd., transl. and notes by ..., Warminster.

Hopkinson, N. 2000: Ovid: Metamorphoses. Book 13, ed. by ..., Cambridge - Nueva York.

Lemaire, N. E. 1822: Publius Ovidius Naso, ex recensione Gott. Erdmann Gierig cum variis lectionibus codicum Parisinorum cui novas addidit notas Nicol. Elig. Lemaire; vol. IV: Met. 8-15, París.

Luck, G. 2009: «More missing letters in Ovid's Metamorphoses», MH 66, pp. 88-119.

Magnus, H. 1914: P. Ovidi Nasonis Metamorphoseon libri XV, Berlín.

Merkel, R. 1850: P. Ovidius Naso, ex recognitione R. Merkelii, vol. II, Leipzig.

Merkel, R. 1875: P. Ovidius Naso, ex iterata R. Merkelii recognitione, vol. II, Leipzig.

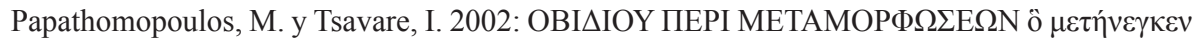

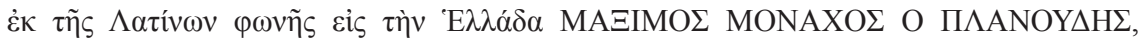
Atenas.

Regius, R. 1493: P. Ouidii Metamorphosis, ed. Raph. Regius, Venecia.

Riese, A. 1872: P. Ovidii Nasonis Carmina, edidit ... vol. II. Editio stereotypa, Leipzig. 
Riese, A. 1889: P. Ovidii Nasonis Carmina, edidit ... vol. II. Editio stereotypa iterum recognita, Leipzig.

Simmons, Ch. 1889: P. Ovidii Nasonis Metamorphoseon XIII. XIV, edited with Introduction, Analysis and Notes by ..., Londres y Nueva York.

Tarrant, R. J. 2004: P. Ouidi Nasonis Metamorphoses, Oxford.

Fecha de recepción de la primera version del artículo: 07/01/2016

Fecha de aceptación: 25/01/2016

Fecha de recepción de la version definitiva: 10/02/2016 